\title{
In-Hospital Cardiac Arrest: Patient Characteristics and Factors Influencing Survival and Neurologic Outcomes
}

\author{
Youngshin Cho \\ Department of Emergency Medicine, Soonchunhyang University Seoul Hospital, Seoul, Korea
}

\begin{abstract}
Objective: We aimed to determine the characteristics of in-hospital cardiac arrest (IHCA) patients, as well as the factors influencing survival to discharge and good neurologic outcome.

Methods: We examined patients who experienced IHCA from January 1, 2011, to December 31, 2013, in Soonchunhyang University Seoul Hospital. They were divided into a survival group and non-survival group. The patient characteristics, including age, sex, comorbid disease, arrest time, arrest location, witnessed arrest, monitoring, arrest cause, arrest rhythm, and cardiopulmonary resuscitation (CPR) duration, were compared between the groups. Moreover, we assessed the factors associated with survival to discharge and good neurologic outcomes by using multivariate logistic regression analysis.

Results: In total, 453 patients of IHCA were observed. The comorbidities in the survival group included neurologic disease $(P<0.001)$, arrhythmia $(P=0.001)$, and myocardial infarction $(P=0.032)$, pneumonia $(P=0.016)$. Other characteristics included cardiac arrest at daytime $(P=0.032)$, cardiogenic arrest cause $(P=0.019)$, and $C P R$ duration $<15$ minutes $(P<0.001)$. The factors associated with survival to discharge included comorbid neurologic disease (odds ratio [OR], 2.191; $\mathrm{P}=0.031$ ), arrhythmia (OR, 3.027; $\mathrm{P}=0.009)$, pneumonia ( $O R, 3.243 ; \mathrm{P}=0.002)$, and $C P R$ duration $<15$ minutes $(O R, 9.638 ; \mathrm{P}<0.001)$. The factors influencing good neurologic outcomes included age $<65$ years $(O R, 3.158 ; P=0.007)$, comorbid disease as arrhythmia ( $O R, 4.921 ; P=0.001)$, pneumonia (OR, 4.551; $\mathrm{P}=0.001)$, hypotension $(\mathrm{OR}, 4.264 ; \mathrm{P}=0.021)$, and $\mathrm{CPR}$ duration $<15$ minutes $(\mathrm{OR}, 6.652 ; \mathrm{P}=0.001)$.

Conclusion: The factors influencing survival to discharge and good neurologic outcomes among IHCA patients included comorbidities, arrest cause, and CPR duration.
\end{abstract}

Keywords: Arrest; Resuscitation; Survival

\section{INTRODUCTION}

In-hospital cardiac arrest (IHCA) is fatal and is associated with a low survival rate. Only a few studies have reported on IHCA incidence in Korea. The Get With The Guideline-Resuscitation Registry of the American Heart Association described the incidence rate of IHCA in the United States since 2000 and indicates that approximately 200,000 patients suffer from IHCA per year [1]. Moreover, the registry stated that survival to hospital discharge improved from $13.7 \%$ in 2000 to $22.3 \%$ in 2009 , and the ratio of poor neurological prognosis decreased from $32.9 \%$ to $28.1 \%$ [2]. A study analyzing the various factors affecting the incidence and survival rate of IHCA could provide information which can in- crease the survival rate and improve the neurological prognosis. Therefore, in the present report, we examined the characteristics of IHCA patients in Soonchunhyang University Seoul Hospital, and identified factors affecting the survival rate and neurological prognosis; based on these results, we also suggest potential measures to improve the survival rate of IHCA patients.

\section{MATERIALS AND METHODS}

In this retrospective observational study, we enrolled IHCA patients aged $>19$ years who suffered from IHCA between January 1 , 2011, and December 31, 2013, in Soonchunhyang University Seoul Hospital. Data were collected from the IHCA registry and medical 
records. Age, sex, underlying disease, arrest time, arrest location, presence or absence of witness or monitoring systems, cause of arrest, initial cardiac arrest rhythm, survival to hospital discharge, and neurological status upon discharge were surveyed from the selected research subjects. The surveyed underlying diseases included neurological disorders, arrhythmia, myocardial infarction, heart failure, diabetes, pneumonia, respiratory failure, hepatic failure, renal failure, hypotension, tumor, multiple traumas, and sepsis. The arrest locations included the intensive care unit (ICU), emergency department, general wards, and other locations (outpatient clinics, operating unit, cardiac catheterization unit, computed tomography [CT] unit, and angiography unit). The neurological status on discharge was evaluated based on the cerebral performance categories (CPC) score. Patients with a CPC value of 1 and 2 were included in the good prognosis group, whereas those with a CPC value of $\geq 3$ were included in the poor prognosis group.

All the statistical analyses were performed using SPSS ver. 14.0 for Windows (SPSS Inc., Chicago, IL, USA). Continuous variables were compared using Student t-test, whereas nominal variables were compared using the chi-square test and Fisher's exact test. In order to analyze the factors affecting survival to hospital discharge and neurological prognosis, multivariate logistic regression analysis was performed using the backward likelihood ratio method. For all the analyses, statistical significance levels were determined at $\mathrm{P}$-values of $<0.05$. The study was approved by the institutional review board of Soonchunhyang University Seoul Hospital (approval no., 2014-09-012), and the informed consent was waived because of the retrospective design of the study.

\section{RESULTS}

\section{Characteristics of the in-hospital cardiac arrest patients}

In total, 486 IHCA patients were examined during the research period; however, two patients aged $<19$ years and 31 patients with a do not attempt resuscitation (DNAR) option were excluded from this study. Among the cases included, we divided the patients into two groups of non-survival group with 388 patients and survival group with 65 patients. Table 1 compares the characteristics of the patients between the survival group and non-survival group; no significant difference was observed in terms of sex and age between these groups.

Comorbidities of neurological disorder $(\mathrm{P}<0.001)$, arrhythmia $(\mathrm{P}=0.001)$, myocardial infarction $(\mathrm{P}=0.032)$, and pneumonia $(\mathrm{P}=$
Table 1. Characteristics of in-hospital cardiac arrest

\begin{tabular}{|c|c|c|c|}
\hline Characteristic & Survival $(n=65)$ & Non-survival $(n=388)$ & P-value \\
\hline Age (yr) & $64.09 \pm 18.03$ & $64.28 \pm 15.13$ & 0.884 \\
\hline Gender & & & 0.276 \\
\hline Male & $34(52.3)$ & $233(60.1)$ & \\
\hline Female & $31(47.7)$ & $155(39.9)$ & \\
\hline \multicolumn{4}{|l|}{ Comorbidities } \\
\hline Neurologic & $26(40.0)$ & $78(20.1)$ & $<0.001$ \\
\hline Arrhythmia & $18(27.7)$ & $47(12.1)$ & 0.001 \\
\hline Myocardial infarction & $16(24.6)$ & $55(14.2)$ & 0.032 \\
\hline Congestive heart failure & $11(16.9)$ & $81(20.9)$ & 0.463 \\
\hline Diabetes & $24(36.9)$ & $117(30.2)$ & 0.273 \\
\hline Pneumonia & $26(40.0)$ & $99(25.5)$ & 0.016 \\
\hline Respiratory insufficiency & $18(27.7)$ & $91(23.5)$ & 0.459 \\
\hline Hepatic insufficiency/failure & $1(1.5)$ & $43(11.1)$ & 0.012 \\
\hline Renal insufficiency/failure & $18(27.7)$ & $142(36.6)$ & 0.164 \\
\hline Hypotension/hypoperfusion & $7(10.8)$ & $20(5.2)$ & 0.077 \\
\hline Hematology/oncology & $12(18.5)$ & $100(25.8)$ & 0.206 \\
\hline Major trauma & $1(1.5)$ & $23(5.9)$ & 0.228 \\
\hline Sepsis & $9(13.8)$ & $113(29.1)$ & 0.010 \\
\hline Initial rhythm & & & 0.068 \\
\hline VFNT & $17(26.2)$ & $65(16.8)$ & \\
\hline PEA, asystole & $48(73.8)$ & $323(83.2))$ & \\
\hline Arrest location & & & $<0.001$ \\
\hline Intensive care unit & $17(26.2)$ & $229(59.0)$ & \\
\hline Emergency department & $13(20)$ & $45(11.6)$ & \\
\hline General ward & $19(29.2)$ & $80(20.6)$ & \\
\hline Others ${ }^{\text {a) }}$ & $16(24.6)$ & $34(8.8)$ & \\
\hline Arrest time & & & 0.032 \\
\hline Day (8:00-18:00) & $39(60.0)$ & $177(45.6)$ & \\
\hline Night (18:01-7:59) & $26(40.0)$ & $211(54.4)$ & \\
\hline Arrest time of the week & & & 0.534 \\
\hline Weekday & $49(75.4)$ & $278(71.6)$ & \\
\hline Weekend & $16(24.6)$ & $110(28.4)$ & \\
\hline Arrest cause & & & 0.019 \\
\hline Presumed cardiac & $29(55.4)$ & $116(29.9)$ & \\
\hline Non cardiac & $36(44.6)$ & $272(70.1)$ & \\
\hline Witnessed & $64(98.5)$ & $374(96.4)$ & 0.707 \\
\hline Monitored & $51(78.5)$ & $327(84.3)$ & 0.243 \\
\hline \multicolumn{4}{|l|}{ Before CPR team arrived } \\
\hline Chest compression & $31(47.7)$ & $224(57.7)$ & 0.131 \\
\hline Ventilation & $25(38.5)$ & $256(66.0)$ & 0.000 \\
\hline Intravenous access & $46(70.8)$ & $313(80.7)$ & 0.068 \\
\hline Medication & $20(30.8)$ & $224(57.7)$ & $<0.001$ \\
\hline Defibrillation & $2(3.1)$ & $20(5.2)$ & 0.754 \\
\hline Intubated state & $23(35.4)$ & $224(57.7)$ & 0.001 \\
\hline CPR duration (min) & & & $<0.001$ \\
\hline $0-15$ & $46(70.8)$ & $158(40.8)$ & \\
\hline $15-35$ & $13(20.0)$ & $90(23.3)$ & \\
\hline$>35$ & $6(9.2)$ & $139(35.9)$ & \\
\hline
\end{tabular}

Values are presented as mean \pm standard deviation or number $(\%)$.

VFNT, ventricular fibrillation/ventricular tachycardia; PEA, pulseless electrical activity; CPR, cardiopulmonary resuscitation.

${ }^{a}$ Outpatient clinic, computed tomography unit, angiography unit, etc. 
$0.016)$ were more frequently observed in the survival group, whereas comorbidities such as hepatic failure $(\mathrm{P}=0.012)$ and sepsis $(\mathrm{P}=0.010)$ were more frequently observed in non-survival group, with a statistically significant difference. The initial rhythm on cardiac arrest was assessed for the presence of ventricular tachycardia, ventricular fibrillation, pulseless electrical activity (PEA), and asystole, but no significant difference in their occurrence was observed between the groups. However, the survival rate differed according to the arrest location (ICU, emergency department, general ward, others; $\mathrm{P}<0.001)$. Although the survival rate increased for arrests occurring during the daytime $(\mathrm{P}=0.032)$, there was no major difference between the survival rate for arrests occurring on weekends and weekdays. Among the causes of cardiac arrest, cardiogenic cause showed a higher survival rate. No significant difference was observed in the survival rate according to the presence or absence of a witness or monitoring systems. The ventilation and medication prior to the arrival of the cardiopulmonary resuscitation (CPR) team were significantly different between the two groups $(\mathrm{P}<0.001$, $\mathrm{P}=0.001$ ); however, the frequency of chest compression, intravenous access, and defibrillation did not differ between the groups. Moreover, the number of patients who underwent tracheal intubation prior to the cardiac arrest and CPR duration significantly differed between the groups ( $\mathrm{P}=0.001, \mathrm{P}<0.001$, respectively). There were 265 cases of CPR discontinuation due to return of spontaneous circulation and 174 cases of CPR discontinuation due to no response. And 13 patients were applied extracorporeal membrane oxygenation and one patient was DNAR signed.

\section{Analysis of factors affecting survival to hospital discharge and good neurological outcome}

To elucidate the factors affecting the survival, we performed multivariate logistic regression analysis and analyzed factors such as sex; age ( $<65$ years); arrest location; causes of cardiogenic arrest; comorbid diseases such as neurological disease, arrhythmia, myocardial infarction, pneumonia, hepatic failure, renal failure, hypotension, and sepsis; arrest time (during day time); initial cardiac arrest rhythm (ventricular fibrillation/ventricular tachycardia); chest compression prior to the arrival of the CPR team; ventilation; intravenous access establishment; drug administration; tracheal intubation; and CPR duration (Table 2).

Compared to arrests in the ICU, arrests at other locations (outpatient clinics, CT unit, angiography unit) were associated with a higher survival rate (odds ratio [OR], 5.888; $\mathrm{P}=0.001$ ). Moreover,
Table 2. Multivariable logistic regression analysis for survival discharge

\begin{tabular}{llc}
\hline Variable & Odds ratio (95\% CI) & P-value \\
\hline $\begin{array}{l}\text { Location (ref: ICU) } \\
\text { Others }\end{array}$ ) & $5.888(2.030-17.081)$ & 0.001 \\
Comorbidity & & \\
Neurologic & $2.191(1.073-4.473)$ & 0.031 \\
Arrhythmia & $3.027(1.316-6.967)$ & 0.009 \\
Pneumonia & $3.243(1.556-6.758)$ & 0.002 \\
Hepatic insufficiency & $0.104(0.012-0.932)$ & 0.043 \\
Sepsis & $0.382(0.155-0.940)$ & 0.036 \\
Before CPR team arrived & & \\
Ventilation & $0.387(0.191-0.784)$ & 0.008 \\
Duration of CPR (ref: $>35$ min) & & \\
0-15 & $9.638(3.626-25.618)$ & $<0.001$ \\
15-35 & $2.795(0.915-8.537)$ & 0.071 \\
\hline
\end{tabular}

Variable included in the logistic regression model: gender, age $<65 \mathrm{yr}$, location (ICU, emergency room, general ward, others), cardiogenic cause, comorbidity (neurologic, arrhythmia, myocardial infarction, pneumonia, hepatic insufficiency, renal insufficiency, hypotension, septicemia, arrest time (day time), initial rhythm (ventricular fibrillation/ventricular tachycardia), before CPR team arrived (chest compression, ventilation, intravenous access, medication), intubation state, and CPR duration (0-15 min, 15-35 min).

$\mathrm{Cl}$, confidence interval; ICU, intensive care unit; CPR, cardiopulmonary resuscitation. ${ }^{a}$ O)utpatient clinic, computed tomography unit, angiography unit, etc.

comorbid diseases such as neurological disorders (OR, 2.191; $\mathrm{P}=$ 0.031), arrhythmia (OR, 3.027; $\mathrm{P}=0.009)$, and pneumonia (OR, 3.243; $\mathrm{P}=0.002$ ) was higher in the survival group. Patients with hepatic insufficiency and sepsis rarely survived $(\mathrm{OR}, 0.104 ; \mathrm{P}=0.043$; $\mathrm{OR}, 0.382 ; \mathrm{P}=0.036$, respectively) and patients who received ventilation prior to the arrival of the CPR team were also more frequently observed in the non-survival group ( $\mathrm{OR}, 0.387 ; \mathrm{P}=0.008$ ). Patients who received CPR for $<15$ minutes showed a higher survival rate than patients who received CPR for $>35$ minutes (OR, 9.638; $\mathrm{P}<0.001)$.

Moreover, we assessed patients with good neurological prognosis after cardiac arrest for factors that might affect the outcome (Table 3 ). The patients aged $<65$ years were 3 times more likely to exhibit good neurological prognosis (OR, 3.158; $\mathrm{P}=0.007)$. Compared to patients who suffered an arrest in the ICU, the patients who suffered an arrest at emergency department (OR, 3.424; $\mathrm{P}=$ 0.031) and other locations (outpatient clinics, CT unit, and angiography unit) showed a better neurological prognosis (OR, 7.035; $\mathrm{P}=0.002$ ). Also, presumed cardiogenic cause for arrest had good neurologic outcome $(\mathrm{OR}, 3.134 ; \mathrm{P}=0.008)$. Patients with comorbid diseases including arrhythmia $(\mathrm{OR}, 4.921 ; \mathrm{P}=0.001)$, pneumonia $(\mathrm{OR}, 4.551 ; \mathrm{P}=0.001)$, and hypotension $(\mathrm{OR}, 4.264 ; \mathrm{P}=0.021)$ ex- 
ChoY • In-Hospital Cardiac Arrest: Survival and Neurologic Outcome Factors

Table 3. Multivariable logistic regression analysis for good neurologic outcome

\begin{tabular}{llc}
\hline Variable & Odds ratio (95\% Cl) & P-value \\
\hline Age $<65$ yr & $3.158(1.372-7.271)$ & 0.007 \\
Location (ref: ICU) & & \\
ER & $3.424(1.117-10.496)$ & 0.031 \\
Others ${ }^{a)}$ & $7.035(2.102-23.539)$ & 0.002 \\
Arrest cause (ref: non-cardiac) & & \\
$\quad$ Cardiac & $3.134(1.343-7.314)$ & 0.008 \\
Comorbidity & & \\
Arrhythmia & $4.921(1.940-12.486)$ & 0.001 \\
Pneumonia & $4.551(1.845-11.225)$ & 0.001 \\
Hypotension & $4.264(1.25-14.544)$ & 0.021 \\
Sepsis & $0.228(0.070-0.750)$ & 0.015 \\
Before CPR team arrived & & \\
Medication & $0.368(0.155-0.873)$ & 0.023 \\
Intubated state & $0.377(0.161-0.880)$ & 0.024 \\
Duration of CPR (ref: $>35$ min) & & \\
0-15 & $6.652(2.270-19.497)$ & 0.001 \\
15-35 & $1.842(0.507-6.698)$ & 0.354 \\
\hline
\end{tabular}

Variable included in the logistic regression model: gender, age $<65 \mathrm{yr}$, location (ICU, ER, general ward, others), presumed cardiogenic cause, comorbidity (arrhythmia, myocardial infarction, pneumonia, hepatic insufficiency, renal insufficiency, hypotension, hematology/oncology, septicemia, arrest time (day time), initial rhythm (ventricular fibrillation/ventricular tachycardia), before CPR team arrived (chest compression, ventilation, intravenous access, medication), intubation state, and CPR duration $<15$ min.

$\mathrm{Cl}$, confidence interval; ref, reference; ICU, intensive care unit; ER, emergency room; $\mathrm{CPR}$, cardiopulmonary resuscitation.

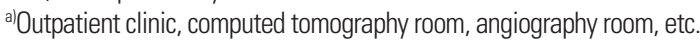

hibited a better neurological outcome. Patient with sepsis, received medication prior to the arrival of the CPR team, and those with tracheal intubation showed a poor neurological prognosis $(\mathrm{OR}$, $0.228 ; \mathrm{P}=0.015 ; \mathrm{OR}, 0.368 ; \mathrm{P}=0.023 ; \mathrm{OR}, 0.377 ; \mathrm{P}=0.024$, respectively). Patients who received CPR for $<15$ minutes showed a better neurological prognosis than patients who received CPR for $>35$ minutes $(\mathrm{OR}, 6.652 ; \mathrm{P}=0.001)$.

\section{DISCUSSION}

Several factors had a significant impact on survival and good neurologic outcome in IHCA patients. These factors included comorbid disease, arrest location, arrest time, arrest cause, CPR performed prior to the arrival of the CPR team, tracheal intubation, and CPR duration.

IHCA patients generally present with various underlying diseases, and the prognosis after cardiac arrest is usually poor. Many researches have reported that the rate of survival to hospital dis- charge in IHCA patients ranges from $15 \%$ to $20 \%$ [3-6]. The presence of underlying diseases influences the survival rate, and certain severe diseases also exhibit low recovery ratio, thus leading to unnecessary in-hospital CPR, wastage of medical resources, and an increase in economic and emotional burden $[7,8]$. The underlying diseases influencing the survival rate in IHCA patients include arrhythmia, myocardial infarction, congestive heart failure, diabetes, pneumonia, respiratory failure, hepatic failure, renal failure, hypotension, hematologic malignancy/solid tumors, multiple traumas, and sepsis [9]. According to the study of Merchant et al. [9], except for congestive heart failure, all the severe underlying diseases reportedly exhibited a significant difference between the survival group and non-survival group; moreover, arrhythmia, myocardial infarction, and diabetes were found to be factors affecting the survival to hospital discharge. Another study examined congestive heart failure, severe respiratory system diseases, hepatic failure, renal failure, hematologic malignancy, and metastatic solid tumors, and found that liver disease, hematologic malignancy, and metastatic solid tumors particularly exhibited a low 6-month survival rate [8]. In the present study, neurological disorders, arrhythmia, myocardial infarction, and pneumonia were more frequently found in the survival group, whereas hepatic failure and sepsis were more frequently found in the non-survival group. We believe that patients with the abovementioned underlying diseases should be informed regarding the possibility of suffering a cardiac arrest and should be prepared for the possible occurrence. Korean hospitals have recently established an emergency response team, and studies have indicated the effectiveness of preventing cardiac arrest of patients with risk factors in advance $[10,11]$.

The initial rhythm on cardiac arrest significantly affects the survival among IHCA patients. Patients with ventricular fibrillation and ventricular tachycardia undergo defibrillation and exhibit a successful therapeutic response, thus leading to an increased survival rate [12]. Moreover, the observation of ventricular fibrillation and ventricular tachycardia as the initial rhythm of IHCA patients might suggest shorter duration from the time of the arrest than PEA and asystole which are usually found as an initial rhythm in case of failure of the initial treatment or delayed recognition of arrest [12]. A study reported that the survival rate of ventricular tachycardia and ventricular fibrillation patients increased from $21 \%$ to $38 \%$ when defibrillation was performed within 3 minutes of the arrest [4]. However, in the present study, the initial cardiac 
arrest rhythm did not show any impact on the survival rate or good neurological prognosis; this may be due to the failure of the appropriate execution of defibrillation on cardiac arrest or delayed defibrillation. A survey revealed that, of 82 patients who exhibited an initial cardiac arrest rhythm that required defibrillation, only $19.9 \%$ received defibrillation before the arrival of the CPR team or attending physician. This may be due to the misconception that defibrillation is a task that should only be attempted by the CPR team or attending physician because of misjudgment of the necessity of defibrillation or due to the lack of confidence of the individual in performing defibrillation. Only $56 \%$ of patients received chest compression before the arrival of the CPR team, whereas $62 \%$ received ventilation by bag valve mask, demonstrating the overall failure of immediate CPR. This finding indicates the lack of an appropriate response to a CPR call in this hospital, which has been subsequently rectified through promoting awareness on this topic.

Following the occurrence of IHCA, an immediate and early response is essential for better survival. However, on weekends and during the night time, an immediate response is less likely. Hence, we believe that the low survival rate during the night time may be due to the relatively inefficient response of the emergency response system, which has also been suggested in other studies [12].

Many studies have indicated that the presence or absence of witness of the IHCA as well as monitoring systems are associated with the survival rate $[3,12,13]$. According to a recent study, cardiac arrest patients in general wards that lack good patient monitoring systems did not exhibit a good recovery [14]. The factors contributing to this finding reportedly include the relatively longer time for detecting arrest than that required in the ICU, as well as the lack of monitoring systems, appropriate equipment, and skilled doctors [3]. Other study indicated that the survival rate of IHCA patients in the ICU is higher because the increased possibility of immediate resuscitation due to good monitoring systems, relatively younger age of the patients, and a relatively lower DNAR ratio in the ICU [12]. However, in this study, no significant difference was observed in the survival rate between the IHCA witness group and non-witness group. This may be due to the lack of a clear recording of what kind of monitoring system was used is measuring oxygen saturation or is recording electrocardiograms, and because monitored ICU patients usually have more severe conditions which lowers the possibility of survival. A higher rate of survival to discharge and better neurological prognosis for patients who suffered an arrest at other locations (outpatient clinics, CT unit, angiogra- phy unit) can also be explained in this manner. Moreover, patients who already underwent tracheal intubation are usually already in very severe conditions, and are therefore likely to show a poor neurological prognosis.

We attempt to improve the outcome of IHCA by predicting the arrest cause prior to the cardiac arrest, maintaining effective CPR, and focusing on post cardiac arrest management after recovery of spontaneous circulation. As previously mentioned, establishing an emergency response team and predicting and preventing the arrest cause prior to the cardiac arrest will be effective measures $[10,11]$. However, maintenance of an effective CPR team would require additional medical resources, such as physician, nurse, equipment, and space, which are limitations for such a measure. Various efforts should be made for effective CPR performance on cardiac arrest. The establishment of a separate professional CPR team is a good strategy for effective CPR [15]. One report has indicated that the survival rate increased by 4 times after nurses were educated in an advanced cardiopulmonary life support (ACLS) course [16]. In our hospital, all nurses a reeducated on basic life support skills; the nurses participate in such sessions every 2 years. Although ACLS course is not yet being provided, we believe that such education is essential for ensuring appropriate initial defibrillation, as nurses are usually the first witness of cardiac arrest. In case with recovery of spontaneous circulation after cardiac arrest, care must be taken to hypothermia, manage blood sugar levels, and prevent infection [12]. Moreover, clearly defining the DNAR status may also be effective in increasing the survival rate of IHCA patients [11]. Cases wherein the patient's DNAR information is not clear before the execution of CPR, or wherein the information is not properly shared, may lead to unnecessary treatment, wastage of medical resources, and medical expenses.

This study has several limitations. Firstly, study was designed retrospectively, so it is difficult to reflect the patients' severity in the analysis. Moreover, it is difficult to consider the sequential organ failure assessment score and Deyo-Charlson score, which may affect the survival rate $[8,15]$. However, the tracheal intubation state and the incidence of cardiac arrest in the ICU may indirectly reflect the severity of the patient's condition. Secondly, we were unable to analyze the manner in which factors such as therapeutic hypothermia after CPR affect the neurological prognosis; this is because our hospital only recently introduced therapeutic hypothermia. A further study that predicts the survival rate and neurological prognosis while considering severity and therapeutic hypo- 
thermia is essential in the near future.

In conclusion, various factors affect the survival rate and good neurological prognosis of IHCA patients. The underlying disease, cause of cardiac arrest, and the time of CPR are particularly relevant. In order to increase the survival of IHCA patients and improve the neurological prognosis, these factors should receive more attention and appropriate education should be provided.

\section{CONFLICT OF INTEREST}

No potential conflict of interest relevant to this article was reported.

\section{REFERENCES}

1. Merchant RM, Yang L, Becker LB, Berg RA, Nadkarni V, Nichol G, et al. Incidence of treated cardiac arrest in hospitalized patients in the United States. Crit Care Med 2011;39:2401-6.

2. Girotra S, Nallamothu BK, Spertus JA, Li Y, Krumholz HM, Chan PS, et al. Trends in survival after in-hospital cardiac arrest. N Engl J Med 2012; 367:1912-20.

3. Chon GR, Lee J, Shin Y, Huh JW, Lim CM, Koh Y, et al. Clinical outcomes of witnessed and monitored cases of in-hospital cardiac arrest in the general ward of a university hospital in Korea. Respir Care 2013;58:1937-44.

4. Peberdy MA, Kaye W, Ornato JP, Larkin GL, Nadkarni V, Mancini ME, et al. Cardiopulmonary resuscitation of adults in the hospital: a report of 14720 cardiac arrests from the National Registry of Cardiopulmonary Resuscitation. Resuscitation 2003;58:297-308.

5. Nadkarni VM, Larkin GL, Peberdy MA, Carey SM, Kaye W, Mancini ME, et al. First documented rhythm and clinical outcome from in-hospital cardiac arrest among children and adults. JAMA 2006;295:50-7.
6. Gwinnutt CL, Columb M, Harris R. Outcome after cardiac arrest in adults in UK hospitals: effect of the 1997 guidelines. Resuscitation 2000; 47:125-35.

7. Heyland DK, Frank C, Groll D, Pichora D, Dodek P, Rocker G, et al. Understanding cardiopulmonary resuscitation decision making: perspectives of seriously ill hospitalized patients and family members. Chest 2006;130: 419-28.

8. Kim SN, Shin TG, Sim MS, Jo IJ, Song HG. Prognosis of in-hospital cardiac arrest and severe comorbidities. J Korean Soc Emerg Med 2010;21: 749-56.

9. Merchant RM, Berg RA, Yang L, Becker LB, Groeneveld PW, Chan PS, et al. Hospital variation in survival after in-hospital cardiac arrest. J Am Heart Assoc 2014;3:e000400.

10. Kwak HJ, Yun I, Kim SH, Sohn JW, Shin DH, Yoon HJ, et al. The extended rapid response system: 1-year experience in a university hospital. J Korean Med Sci 2014;29:423-30.

11. Shin TG, Jo IJ, Song HG, Sim MS, Song KJ. Improving survival rate of patients with in-hospital cardiac arrest: five years of experience in a single center in Korea. J Korean Med Sci 2012;27:146-52.

12. Sandroni C, Nolan J, Cavallaro F, Antonelli M. In-hospital cardiac arrest: incidence, prognosis and possible measures to improve survival. Intensive Care Med 2007;33:237-45.

13. Brady WJ, Gurka KK, Mehring B, Peberdy MA, O'Connor RE; American Heart Association's Get with the Guidelines (formerly, NRCPR) Investigators. In-hospital cardiac arrest: impact of monitoring and witnessed event on patient survival and neurologic status at hospital discharge. Resuscitation 2011;82:845-52.

14. Schwartz BC, Jayaraman D, Warshawsky PJ. Survival from in-hospital cardiac arrest on the internal medicine clinical teaching unit. Can J Cardiol 2013;29:117-21.

15. Park HA, Kang MJ, Cha WC, Shin TG, Jo IJ, Song KJ, et al. Effectiveness of a cardiopulmonary resuscitation team with an emergency physician for in-hospital cardiac arrest. J Korean Soc Emerg Med 2012;23:603-10.

16. Dane FC, Russell-Lindgren KS, Parish DC, Durham MD, Brown TD. Inhospital resuscitation: association between ACLS training and survival to discharge. Resuscitation 2000;47:83-7. 